\title{
$1 \quad$ Naa15 knockdown enhances c2c12 myoblast fusion and induces 2 defects in zebrafish myotome morphogenesis
}

3 Olivier Monestier ${ }^{1 \$ *}$, Aurélie Landemaine ${ }^{1}$, Jérôme Bugeon ${ }^{1}$, Pierre-Yves Rescan ${ }^{1}$, Jean-Charles 4 Gabillard ${ }^{1 \mathrm{~s}}$

$5{ }^{1}$ INRA, UR1037 Laboratoire de Physiologie et Génomique des Poissons, Campus de Beaulieu, 635000 Rennes, FRANCE.

$7 \quad{ }^{\$}$ Corresponding authors: monestierolivier@yahoo.fr; jean-charles.gabillard@inra.fr

$8{ }^{*}$ Present address for Olivier Monestier: IRIBHM, ULB, Route de Lennik 808, BtC, Local C6-140, 1070 Bruxelles, 9 Belgium. 


\section{Abstract}

11 The comprehension of muscle tissue formation and regeneration is essential to develop

12 therapeutic approaches against muscle diseases or loss in muscle mass and strength during

13 ageing or cancer. One of the critical steps in muscle formation is the fusion of muscle cells to

14 form or regenerate muscle fibres. To identify new genes controlling myoblast fusion, we

15 undertook an siRNA screen in c2c12 myoblasts and found that $\mathrm{N}$-alpha-acetyltransferase 15

16 (Naa15) knockdown enhanced c2c12 myoblast fusion suggesting that Naa15 negatively

17 regulated myogenic cell fusion. We identified two Naa15 orthologous genes in zebrafish

18 genome: naa15a and naa15b. These two orthologs are both expressed in myogenic domain of

19 the somite. Knockdown of zebrafish naal5a and naal5b genes induced a "U" shaped

20 segmentation of the myotome and alteration of myotome boundaries resulting in the

21 formation of abnormally long myofibres spanning adjacent somites. Taken together these

22 results show that Naa15 regulates myotome formation and myogenesis in fish.

\section{$23 \quad$ Keywords}

24 siRNA screen, morpholino, trout, muscle regeneration, evolution

\section{$25 \quad$ 1. Introduction}

26 The fusion process is a critical step for the formation and reparation of muscle. Indeed,

27 skeletal muscle is mainly composed of multinucleated cells, called myofibres. To form those

28 myofibres, progenitor cells (myoblast) proliferate, differentiate into myocytes, fuse to form

29 multinucleated myotubes and finally mature into functional myofibres. The process leading to

30 the formation of skeletal muscle is usually divided into a primary, a secondary, and an adult 
31 phases (1). The primary phase is powered by cells originating from dermomyotome lips and

32 results in the formation of the primary myotome. Then, during the secondary phase, cells

33 emanating from the central region of the dermomyotome, differentiate and fuse either with

34 each other to form secondary fibres (hyperplasic growth) or with primary fibres (hypertrophic

35 growth). Through adult phase, satellite cells allow muscle growth, and regeneration after

36 damage and injury.

Myocyte fusion is a very coordinated event requiring that two cells get close, recognize

38 each other, adhere their membrane, open fusion pore and finally merge together into one

39 multinucleated cell (2). This process implies many known molecular components studied in

40 multiple model organisms like drosophila, zebrafish and mouse.

41 In the fruit fly Drosophila melanogaster, each muscle is composed of a single myofibre

42 formed by the fusion of a unique founder cell (FC) with fusion competent myocyte (FCM).

43 Recognition and adhesion between FC and FCM are mediated by immunoglobulin domain

44 containing cell adhesion molecules. Among them, are Kin of IrreC (Kirre) and Roughest (Rst)

45 which are expressed in FC $(3,4)$ as well as ticks and stones (Sns) and Hibris (Hbs) both

46 expressed in FCM (5-8). In vertebrate species the presence of two types of muscle cells has

47 not yet been demonstrated, nevertheless, some genes initially identified in drosophila have

48 homologs in vertebrates. For example Kirrel (Kirre homologue) and nephrin (Sns homologue)

49 are both involved in cell recognition/adhesion process $(9,10)$. The fusion of myocytes in

50 vertebrates also implies specific factors such as myomaker, myomerger and myomixer (11-

51 15) and some, species-specific factors such as Jamb and Jamc in zebrafish (16) or Itgb1 in

52 mouse (17).

53 After the initial recognition/adhesion step, greater membrane proximity is required and

54 reached by a reorganization of actin cytoskeleton. This is achieved, in Drosophila, by 
55 regulators like WASP and Scar that affect actin polymerization mediated by the Arp2/3

56 complex (18-22). In vertebrate, this step is dependent of Dock1 and Dock5 (23,24), Rac1

57 (23,25), and N-WASP (26).

58 At last the lipid bilayer needs to be destabilized to allow the cells to merge. Some studies

59 performed in c2c12 cells and in chicken show that the family of brain angiogenesis inhibitor

60 molecules (BAI) play a major role during this step of the myocyte fusion (27-29).

61 The regulation of myocytes fusion is highly complex and is far to be completely

62 understood. To identify new genes implicated in the myocyte fusion process in vertebrates, we

63 performed an in vitro functional screen in c2c12 cell line based on siRNA knockdown. We

64 found that Naa15 knockdown led to the formation of myotube larger than those found in

65 c2c12 control cells. In line with this observation, knockdown of the two zebrafish orthologous

66 genes naa15a and naal5b induced the production of giant myofibres spanning two somites in

67 zebrafish embryos suggesting that Naa15 negatively regulated myofibre formation.

\section{2. Materials and Methods}

\section{Zebrafish husbandry}

70 Wild-type zebrafish (Danio rerio) were raised in our facilities (INRA LPGP, Rennes) and

71 maintained under oxygen saturation in a recirculating water system at $27 \pm 1{ }^{\circ} \mathrm{C}, \mathrm{pH} 7.5$.

72 Zebrafish were exposed to a photoperiod of $16 \mathrm{~h}$ light $/ 8 \mathrm{~h}$ dark. Fish used in this study were

73 reared and handled in strict accordance with French and European policies and guidelines of

74 the Institutional Animal Care and Use Committee (DDSV approval \#35-47). 
c2c12-MCK:GFP mouse myoblast cells (ATCC-CRL-1772 modified) were maintained at

$7737^{\circ} \mathrm{C}$ and seed in 96 wells plates at a density of 10.000 cells/well in Growth Medium

78 (Dulbecco's modified Eagle's medium (DMEM), supplemented with 10\% heat-inactivated

79 fetal bovine serum (FBS) and Antibiotic-Antimycotic solution (BIOWEST)). Two hundred

80 fifty five genes expressed in proliferating or differentiating C2C12 cells (Moran et al 2002;

81 Tomczack et al., 2003) but with unknown function, were tested in this screen. Each gene was

82 knocking down with two different siRNA (Flexiplate siRNA, Quiagen). Each plate included

83 two negative controls (no siRNA) and two positive controls treated with anti IL4 siRNA. The

84 cells were transfected at day 1 and day 4 with DMEM including $5 \mathrm{nM}$ of siRNA and $1 \mu 1$ per

85 well of INTERFERin (Polyplus) and placed in differentiation medium (DMEM containing

$862 \%$ FBS and Antibiotic-Antimycotic solution). The medium was changed every day. After six

87 days of differentiation, cells were washed two times with phosphate-buffered saline (PBS)

88 before fixing in PBS with 4\% paraformaldehyde for $15 \mathrm{~min}$. Then, the cells were

89 permeabilized 3 minutes in PBS with $0.1 \%$ TritonX100, stained with $0.1 \mu \mathrm{g} / \mathrm{ml}$ of DAPI for 5

90 minutes and stored at $4^{\circ} \mathrm{C}$ in the dark.

91 Image acquisition was made using an HCS Arrayscan VTI (Cellomics/Thermofisher

92 Scientific) with a ZEISS EC plan NEOFluar 10x ON objective and an ORCA-ER 1.00

93 camera. A macrocommand was edited for visilog 6.7 to monitor automatically the fusion

94 index, mean nuclei number per GFP+ cells, and the GFP+ cells area.

\section{Cell culture for QPCR analysis}

96 The c2c12-MCK:GFP mouse myoblast cells were maintained at $37^{\circ} \mathrm{C}$ and seeded in 12

97 wells plates at a density of 100.000 cells/well in growth medium. Naa15 was knocking down

98 with two different siRNA (Flexiplate siRNA, Quiagen). The cells were transfected at day 1

99 with DMEM including 5nM of siRNA and 3.5 $\mu 1$ per well of INTERFERin (Polyplus) and 
place in differentiation medium. Each plate includes 4 wells transfected with anti-GFP siRNA

101 as negative control, and 4 wells transfected with each of the 2 anti-Naa15 siRNA. The

102 medium was changed every day. One plate was stopped after $0,1,2$ or 3 days of

103 differentiation. Total RNA was isolated using TRIzol (Invitrogen) according to the

104 manufacturer's instructions and relative RNA concentration was determined by

105 spectrophotometric analysis. $0.3 \mu \mathrm{g}$ of total RNA was reverse-transcribed into cDNA using

106 the high-capacity cDNA reverse transcription kit (Applied Biosystems). Realtime PCR was

107 performed in duplicate using $1 / 10,1 / 40$ or $1 / 400$ dilution of RT-cDNA from c2c12 cells.

108 Samples were amplified in a 96 well plate using SYBR Green on a StepOnePlus Real Time

109 PCR system (Applied Biosystems) with specific primers for mouse Naa15, and Myog genes at

110 a concentration of $300 \mathrm{nM}$. The expression of Hprt and B-Actin genes were used as

111 endogenous control to normalize each sample. Relative mRNA expression was assessed by

112 relative standard curve method.

114 In situ hybridization

115 Embryos were removed from their chorion by a $3 \mathrm{~min}$ incubation in a 1/100 (wt/vol)

116 Pronase solution (Sigma, P6911) pre-warmed to $28{ }^{\circ} \mathrm{C}$. Then, they were fixed in $4 \%$

117 paraformaldehyde overnight and stored in methanol at $-20{ }^{\circ} \mathrm{C}$. Anti-sense RNA probes

118 labelled with digoxigenin (DIG) were prepared from PCR-amplified templates using

119 appropriate RNA polymerases. Whole mount in situ hybridization were performed with an

120 INSITU PRO VS automate (INTAVIS AG) using standard protocol (30). Whole mount in

121 situ images were obtained using a macroscope NIKON AZ 100 coupled with NIKON Digital

122 Sight DSRil camera and using NIS-Elements D 3.2 software. 


\section{Morpholino injection}

125 Freshly fertilized eggs were injected with morpholinos at one to two-cell stage.

126 Morpholinos (Gene Tools) were dissolved in sterile water at a concentration of $2.5 \mathrm{mM}$. Anti-

127 naa15a, anti-naa15b, anti-p53 and anti-naa15a mismatch control morpholinos were used.

128 Anti-Naa15a and anti Naa15b were designed to bind the area of the predicted start codon.

129 Morpholinos sequences were as follows: anti-naal5a:

130 TCTTGAGGGTTGTCCACCGCGACTT; anti-naal5b:

131 CGGCATCCTGTTCACTCTCTATTTC; anti-naa15a mismatch control:

132 TATTGAGGGTTGT $\underline{A C A C C} \underline{C} C G A \underline{A}$ TT. 300 to 450 eggs were injected for each experiment.

133 Embryos were injected with approximately $4 \mathrm{nl}$ of morpholinos ( $8.8 \mathrm{ng})$ diluted in sterile

134 water with $0.1 \%$ phenol red. About the same number of eggs was injected with the anti-

135 naa15a mismatch control. To ensure that phenotype specificity is due to knock down of

136 Naa15 orthologues and not to nonspecific induction of apoptosis, embryos were co-injected

137 with anti-p53 morpholino (6.5ng of anti-naa15a+6.5ng of anti-p53). The injected eggs were

138 cultured at $28{ }^{\circ} \mathrm{C}$, and embryos were fixed in $4 \%$ paraformaldehyde overnight at $30 \mathrm{~h}$ of

139 development. Zebrafish embryos were permeabilized in $0.3 \%$ Triton in PBS solution for $3 \mathrm{~h}$.

140 Embryos were stained with a solution including $5 \mu \mathrm{g} / \mathrm{ml}$ of WGA-Alexa 488 and TOPRO 3

141 (1:1000 dilution) overnight. Confocal microscopy images were collected using an OLYMPUS

142 BX61WI FV 1000 microscope and FluoView 3.0 software. Entire images were adjusted for

143 contrast, brightness, and dynamic range using ImageJ software.

\section{Statistical analyses}


For the siRNA screen, statistical analysis was made using a One-way ANOVA (ANalysis

147 Of Variance) with post-hoc Tukey test in SigmaStat 3.5. For QPCR experiment statistical 148 analysis was made using a One-way ANOVA in Past 3.15.

\section{Results}

\section{Naa15 knockdown enhanced c2c12 myoblast fusion}

152 An in vitro functional screen was performed based on siRNA knockdown (KD) in

153 MCK:GFP C2C12 myoblasts. Those myoblasts express GFP under the muscle creatine kinase

154 (MCK) promoter, GFP is therefore expressed only in differentiated myoblasts allowing an

155 easy monitoring of cells differentiation. Our functional screening allowed the identification of

156 genes which in vitro knock-down significantly impacted myoblast fusion without affecting the

157 differentiation capacity of the cells, various functional parameters were assessed among which

158 the fusion index, mean nuclei number per GFP+ cells, and the GFP+ cells area.

159 One of the more strong phenotype was observed after the KD of the $\mathrm{N}$ (Alpha)-

160 Acetyltransferase 15 (Naa15 also referred to as Tbdn, Narg1, mNat1, NATH) gene (Fig 1A).

161 The c2c12 cells transfected with two different anti-Naa15 siRNA exhibited a significant

162 increase in the fusion index. This index (number of nuclei in myotubes / total number of

163 nuclei) was around 75\% greater in GFP+ (differentiated) cells after treatment with both

164 Naa15 siRNA than in control cells (Fig 1B). Specifically, the mean number of cells including

165 more than 10 nuclei after 7 days of differentiation was five times higher for cell cultures

166 treated with anti-Naal5 siRNA when compared to control cell cultures. No significant

167 difference was observed in the number of small myotubes ( $<4$ nuclei) (Fig 1C). 
QPCR experiment showed that both siRNA treatment induced a 50\% reduction of Naal5

169 expression (Fig 1D). As shown by differentiation index, no significant changes in the

170 expression of Myogenin $(M y o G)$, a differentiation marker, was observed in the cells

171 transfected with anti-Naa15 siRNA as compare to controls (Fig 1E). This confirm that the

172 differentiation process was not impacted by Naa15 knockdown.

175 zebrafish somites

176 To study the function of naa15 during myogenesis in zebrafish, we looked for orthologous

177 genes. We performed BLAST in public databases and identified two orthologous genes

178 namely naa $15 a$ and naa $15 b$. Those genes encode proteins with respectively $85 \%$ and $90 \%$ of

179 homology with the murine NAA15 protein. Protein sequences were used to deduce a

180 phylogenetic tree from maximum likelihood method (Fig 2) and show an naa15 duplication

181 occurring in teleost and in cyprinid as expected.

182 In situ hybridization showed that naal5a (Fig 3A) and naal5b (Fig 3B) were both

183 expressed in somites during late somitogenesis ( $24 \mathrm{hpf})$, when myoblast fusion process

184 occurs. There are also expressed in eyes and midbrain. It could be noticed that only a weak

185 signal was observed for naa15b in situ hybridization. 
To determine whether naal5a and naal5b are required for fish myogenesis in vivo, we

190 performed morpholino knockdown of naa15a and naa15b. Zebrafish embryos at 1 cell stage

191 were injected with either an anti-naa15a, an anti-naa15b, or an anti-naa15a mismatch

192 morpholino (4 mismatches) used as a control. The control embryos injected with anti-naa15a

193 mismatch morpholino exhibited a normal morphology at 30hpf and no phenotype was

194 observed as compare to wild type uninjected embryos. At $30 \mathrm{hpf}$, naa15a or naal5b morphant

195 exhibited morphological defect that could be classified in two classes. The class 1 embryos

196 (C1) had reduction of the body size and small curvature of the trunk. The phenotype of the

197 class 2 embryos (C2) is more severe, and a greater curvature of the trunk is observed (Fig 4).

198 According to the experiment, the $\mathrm{C} 1$ embryos represent $30-50 \%$ of the naa $15 a$ and $15-25 \%$ of

199 the naal5b morphants, and the $\mathrm{C} 2$ embryos represent $30-50 \%$ of the naa $15 \mathrm{a}$ and $40-56 \%$ of

200 the naa15b morphants. Wild type phenotype is observed in $5-20 \%$ of the naa15a morphants

201 and in $20-30 \%$ of the naal $5 b$ morphants.

202 Co-injection with anti-naa15a and anti-naa15b morpholino was also performed. Most of

203 the embryos co-injected with the two morpholino died in the first 24 hours after fertilization.

204 The survivors naa15a naal5b morphants presented similar phenotypes to embryos injected

205 with anti-naa15a or anti-naa15b alone (C1 and C2 phenotypes).

206 To confirm that the observed phenotypes did not result from unspecific induction of

207 apoptosis (31), the experiments were replicated with embryos co-injected with anti-naa15a

208 and anti-p53 morpholinos. In all experiments, the naa15a/p53 morphants present the same

209 phenotype than the embryos injected with anti-naa15a or anti-naa15b morpholinos alone. 


\section{2 intersegmental boundary defect}

213 Confocal microscopy of the morphants after staining of the nuclei and the plasma

214 membrane and connective tissue revealed defect in the segmentation of the $\mathrm{C} 1$ and $\mathrm{C} 2$

215 embryos as compare to control mismatch naa15 morphants. Wheat Germ Agglutinin (WGA)

216 conjugate with alexa488 was used for the plasma membrane and connective tissue staining.

217 This protein is a lectin that selectively binds to $\mathrm{N}$-acetylglucosamine and $\mathrm{N}$-acetylneuraminic

218 acid (sialic acid) residues and allows fast, and convenient methodology for connective tissue,

219 and plasma membrane visualization (32). This methodology allowed us to shown that $\mathrm{C} 1$ and

$220 \mathrm{C} 2$ embryos did not exhibit the usual chevron shape segmentation of the myotomes (Fig 5)

221 (33). Further, the myotome of the naal5a and naal5b morphants presented a lack or

222 reduction of the horizontal myoseptum and interruption of myotome boundaries along the

223 dorso/ventral and medio/lateral axis (Fig 5). Those interruptions were observed in all the

224 tested $\mathrm{C} 1$ and $\mathrm{C} 2$ embryos $(\mathrm{n}>15)$ in about $30 \%$ of the myotome boundaries. They formed

225 holes through which some of the myofibres, displaying twice the normal fibre length,

226 stretched out (figure 5, figure 6). Some of those longer myofibres could contain up to 11

227 nuclei but altogether, their mean number of nuclei per fibres was not significantly increased as

228 compared to normal size fibres (not shown).

\section{9 naa15a and naa15b knockdown in zebrafish led to a}

\section{0 reorganization of the myosin pattern}

231 In the $\mathrm{C} 1$ an $\mathrm{C} 2$ naal5a and naal5b morphants, the myosin proteins had not the same

232 expression pattern than in the wild type embryos, as shown by myosins immunostaining

233 performed using MF20 antibody. Myosin is mainly located at the peripheries of the wildtype

234 control embryos myofibres, close to the myotome boundary (Fig 6A, E) whereas in the C1 
235 and C2 morphants, it was uniformly present within the whole fibre (Fig 6B, F). This abnormal

236 myosin localization was also observed in long fibre spanning the intersegmental boundaries 237 (Fig 6B, F).

\section{Discussion}

239 The fusion of muscle progenitor cells is one of the critical steps in muscle formation and

240 regeneration. This process requires several steps including cell recognition, adhesion, and

241 membrane fusion. To identify new genes implicated in myocyte fusion in vertebrates, we

242 undertook an siRNA screen in c2c12 myoblasts followed by in vivo functional studies of

243 relevant candidate genes in zebrafish.

244 Among the tested genes, we identified Naa15 as an inhibitor of c2c12 fusion since its

245 knockdown enhanced fusion of myoblasts in vitro. Naa15 is part of the family of N-terminal

246 acetyltransferase subunits. The NAA15 protein is highly expressed during embryogenesis

247 (34-36) and it binds to the catalytic subunit Naa10 to form the NatA complex (for review of

248 NAT complex see (36)). In mammals, the NatA complex interact with various substrates and

249 is implicated in a broad range of cellular processes from cell growth to cellular differentiation

250 (35-44). The major function of the NatA complex is the proteins N-terminal acetylation (36).

251 The N-terminal acetylatation has various consequence for a protein: it could determine the

252 subcellular localization (45-48), module the protein-protein interactions $(49,50)$ and is also

253 crucial for protein folding. Neither Naa15 or the NatA complex is currently described to have

254 a function during myogenesis. Nevertheless, other N-terminal acetyltransferase was reported

255 to play a key role in tropomyosin-actin complex formation, increasing actin binding, and

256 promoting the regulation of specific myosin activity $(51,52)$. 
Our in silico analysis revealed that the Naa15 gene is found in two copies (naa15a and

258 naa15b) in the zebrafish genome as a result of the Teleost Genome Duplication (TGD) (53).

259 In situ hybridization analysis indicated that naa15a and naa15b were expressed in somites at

$26024 \mathrm{~h}$ post fecundation, when myoblast fusion occurred (16). To further assess the role of

261 Naa15 in myogenesis, we undertook knockdown experiments in zebrafish using morpholinos.

262 We observed that zebrafish embryos injected with anti naa15 Morpholinos didn't exhibit

263 classical chevron-shaped myotomes. Further, interruptions in the intersegmental boundaries

264 allowing long myofibres to span over two (rarely 3) segments. This phenotype is reminiscent

265 with those observed after the knockdown of genes encoding components of the Notch

266 signalling pathway, especially herl and her7 (54,55).

267 NAA15 is known to be a binding partner of cortactin [31], a protein regulating the F-actin

268 polymerization and as such could be involved in process such as migration, permeability or

269 elongation of cells. The knockdown of Naa15 in retinal endothelial cells induces activation of

270 the c-SRC kinase resulting in the phosphorylation and activation of the cortactin by a still

271 unknown mechanism (39). This activation of cortactin resulting of the naa15a or naa15b

272 knockdown could be partially responsible of the phenotype we observed. Indeed, increasing

273 cell permeability, adhesion and migration could lead to the in vitro enhancement of myoblast

274 fusion, and the presence of longer myofibres in vivo. Nevertheless, we did not detect any

275 significant modifications in cells migration or adhesion after Naa15 knockdown (not shown).

276 In conclusion our results showed that Naa15 not only inhibits c2c12 myoblast fusion in

277 vitro, but also is expressed in zebrafish developing myotome where it appears to be essential

278 for proper myotome formation. Further research is needed to decipher the possible functional

279 link between Naa15 activity and the notch pathway or the cortactin activity that could explain

280 the phenotype of zebrafish embryos injected with anti naal5 morpholinos. Altogether the 
281 better understanding of the acetylation process leading to the formation and reparation of

282 muscle fibres will be useful to enhance muscle repair therapy.

283

284

285

286

287

288

289

290

\section{Acknowledgments}

This project and O. Monestier fellowship were supported by the French National Research Agency (ANR-12-JSV7-0001-01). The fellowship of Aurélie Landemaine was supported by INRA PHASE and the Région Bretagne. The funders had no role in study design, data collection and analysis, decision to publish, or preparation of the manuscript. We also thank
A. Patinote for zebrafish husbandry and the supply of eggs for microinjection and R Le Guével for automatic acquisition of pictures on the ImPACcell platform (http://imageriepuces-a-cellules.univ-rennes1.fr).

\section{References}

1. Tajbakhsh S. Skeletal muscle stem cells in developmental versus regenerative myogenesis. J Intern Med. 2009 Oct;266(4):372-89.

2. Rochlin K, Yu S, Roy S, Baylies MK. Myoblast fusion: when it takes more to make one. Dev Biol. 2010 May 1;341(1):66-83.

3. Strünkelnberg M, Bonengel B, Moda LM, Hertenstein A, de Couet HG, Ramos RG, et al. rst and its paralogue kirre act redundantly during embryonic muscle development in Drosophila. Development. 2001 Nov;128(21):422939.

4. Ruiz-Gómez M, Coutts N, Price A, Taylor MV, Bate M. Drosophila dumbfounded: a myoblast attractant essential for fusion. Cell. 2000 Jul 21;102(2):189-98.

5. Bour BA, Chakravarti M, West JM, Abmayr SM. Drosophila SNS, a member of the immunoglobulin superfamily that is essential for myoblast fusion. Genes Dev. 2000 Jun 15;14(12):1498-511.

6. Dworak HA, Charles MA, Pellerano LB, Sink H. Characterization of Drosophila hibris, a gene related to human nephrin. Development. 2001 Nov;128(21):4265-76.

7. Artero RD, Castanon I, Baylies MK. The immunoglobulin-like protein Hibris functions as a dose-dependent regulator of myoblast fusion and is 
differentially controlled by Ras and Notch signaling. Development. 2001 Nov;128(21):4251-64.

313 8. Shelton C, Kocherlakota KS, Zhuang S, Abmayr SM. The immunoglobulin superfamily member Hbs functions redundantly with Sns in interactions between founder and fusion-competent myoblasts. Development. 2009 Apr;136(7):1159-68.

9. Srinivas BP, Woo J, Leong WY, Roy S. A conserved molecular pathway mediates myoblast fusion in insects and vertebrates. Nat Genet. 2007 Jun;39(6):781-6.

10. Sohn RL, Huang P, Kawahara G, Mitchell M, Guyon J, Kalluri R, et al. A role for nephrin, a renal protein, in vertebrate skeletal muscle cell fusion. Proc Natl Acad Sci USA. 2009 Jun 9;106(23):9274-9.

11. Millay DP, O'Rourke JR, Sutherland LB, Bezprozvannaya S, Shelton JM, BasselDuby $\mathrm{R}$, et al. Myomaker is a membrane activator of myoblast fusion and muscle formation. Nature. 2013 Jul 18;499(7458):301-5.

12. Landemaine A, Rescan P-Y, Gabillard J-C. Myomaker mediates fusion of fast myocytes in zebrafish embryos. Biochem Biophys Res Commun. 2014 Sep 5;451(4):480-4.

13. Quinn ME, Goh Q, Kurosaka M, Gamage DG, Petrany MJ, Prasad V, et al. Myomerger induces fusion of non-fusogenic cells and is required for skeletal muscle development. Nat Commun. 2017 Jun 1;8:15665.

14. Bi P, Ramirez-Martinez A, Li H, Cannavino J, McAnally JR, Shelton JM, et al. Control of muscle formation by the fusogenic micropeptide myomixer. Science. 2017 21;356(6335):323-7.

15. Zhang W, Roy S. Myomaker is required for the fusion of fast-twitch myocytes in the zebrafish embryo. Dev Biol. 2017 01;423(1):24-33.

16. Powell GT, Wright GJ. Jamb and jamc are essential for vertebrate myocyte fusion. PLoS Biol. 2011 Dec;9(12):e1001216.

17. Schwander M, Leu M, Stumm M, Dorchies OM, Ruegg UT, Schittny J, et al. Betal integrins regulate myoblast fusion and sarcomere assembly. Dev Cell.

18. Massarwa R 'ada, Carmon S, Shilo B-Z, Schejter ED. WIP/WASp-based actinpolymerization machinery is essential for myoblast fusion in Drosophila. Dev Cell. 2007 Apr;12(4):557-69.

19. Richardson BE, Beckett K, Nowak SJ, Baylies MK. SCAR/WAVE and Arp2/3 are crucial for cytoskeletal remodeling at the site of myoblast fusion. Development. 2007 Dec;134(24):4357-67.

20. Schäfer G, Weber S, Holz A, Bogdan S, Schumacher S, Müller A, et al. The Wiskott-Aldrich syndrome protein (WASP) is essential for myoblast fusion in Drosophila. Dev Biol. 2007 Apr 15;304(2):664-74. 
21. Kim S, Shilagardi K, Zhang S, Hong SN, Sens KL, Bo J, et al. A critical function for the actin cytoskeleton in targeted exocytosis of prefusion vesicles during myoblast fusion. Dev Cell. 2007 Apr;12(4):571-86.

22. Gildor B, Massarwa R 'ada, Shilo B-Z, Schejter ED. The SCAR and WASp nucleation-promoting factors act sequentially to mediate Drosophila myoblast fusion. EMBO Rep. 2009 Sep;10(9):1043-50.

23. Moore CA, Parkin CA, Bidet Y, Ingham PW. A role for the Myoblast city homologues Dock1 and Dock5 and the adaptor proteins Crk and Crk-like in zebrafish myoblast fusion. Development. 2007 Sep;134(17):3145-53.

24. Laurin M, Fradet N, Blangy A, Hall A, Vuori K, Côté J-F. The atypical Rac activator Dock180 (Dock1) regulates myoblast fusion in vivo. Proc Natl Acad Sci USA. 2008 Oct 7;105(40):15446-51.

25. Vasyutina E, Martarelli B, Brakebusch C, Wende H, Birchmeier C. The small Gproteins Rac1 and Cdc42 are essential for myoblast fusion in the mouse. Proc Natl Acad Sci USA. 2009 Jun 2;106(22):8935-40.

26. Gruenbaum-Cohen Y, Harel I, Umansky K-B, Tzahor E, Snapper SB, Shilo B-Z, et al. The actin regulator $\mathrm{N}$-WASp is required for muscle-cell fusion in mice. Proc Natl Acad Sci USA. 2012 Jul 10;109(28):11211-6.

27. Hochreiter-Hufford AE, Lee CS, Kinchen JM, Sokolowski JD, Arandjelovic S, Call JA, et al. Phosphatidylserine receptor BAll and apoptotic cells as new promoters of myoblast fusion. Nature. 2013 May 9;497(7448):263-7.

28. Park D, Tosello-Trampont A-C, Elliott MR, Lu M, Haney LB, Ma Z, et al. BAIl is an engulfment receptor for apoptotic cells upstream of the ELMO/Dock180/Rac module. Nature. 2007 Nov 15;450(7168):430-4.

29. Hamoud N, Tran V, Croteau L-P, Kania A, Côté J-F. G-protein coupled receptor BAI3 promotes myoblast fusion in vertebrates. Proc Natl Acad Sci USA. 2014 Mar 11;111(10):3745-50.

30. Thisse $C$, Thisse B. High-resolution in situ hybridization to whole-mount zebrafish embryos. Nat Protoc. 2008;3(1):59-69.

31. Bedell VM, Westcot SE, Ekker SC. Lessons from morpholino-based screening in zebrafish. Brief Funct Genomics. 2011 Jul;10(4):181-8.

32. Kostrominova TY. Application of WGA lectin staining for visualization of the connective tissue in skeletal muscle, bone, and ligament/tendon studies. Microsc Res Tech. 2011 Jan;74(1):18-22.

33. Rost F, Eugster C, Schröter C, Oates AC, Brusch L. Chevron formation of the zebrafish muscle segments. J Exp Biol. 2014 Nov 1;217(Pt 21):3870-82.

34. Gendron RL, Adams LC, Paradis H. Tubedown-1, a novel acetyltransferase associated with blood vessel development. Dev Dyn. 2000 Jun;218(2):30015. 
35. Sugiura N, Adams SM, Corriveau RA. An evolutionarily conserved N-terminal acetyltransferase complex associated with neuronal development. J Biol Chem. 2003 Oct 10;278(41):40113-20.

36. Kalvik TV, Arnesen T. Protein N-terminal acetyltransferases in cancer. Oncogene. 2013 Jan 17;32(3):269-76.

37. Gendron RL, Good WV, Adams LC, Paradis H. Suppressed expression of tubedown-1 in retinal neovascularization of proliferative diabetic retinopathy. Invest Ophthalmol Vis Sci. 2001 Nov;42(12):3000-7.

38. Paradis H, Liu C-Y, Saika S, Azhar M, Doetschman T, Good WV, et al. Tubedown-1 in remodeling of the developing vitreal vasculature in vivo and regulation of capillary outgrowth in vitro. Dev Biol. 2002 Sep 1;249(1):14055.

39. Paradis H, Islam T, Tucker S, Tao L, Koubi S, Gendron RL. Tubedown associates with cortactin and controls permeability of retinal endothelial cells to albumin. J Cell Sci. 2008 Jun 15;121(Pt 12):1965-72.

40. Geissenhöner A, Weise C, Ehrenhofer-Murray AE. Dependence of ORC silencing function on NatA-mediated Nalpha acetylation in Saccharomyces cerevisiae. Mol Cell Biol. 2004 Dec;24(23):10300-12.

41. Wall DS, Gendron RL, Good WV, Miskiewicz E, Woodland M, Leblanc K, et al. Conditional knockdown of tubedown-1 in endothelial cells leads to neovascular retinopathy. Invest Ophthalmol Vis Sci. 2004 Oct;45(10):370412.

42. Wang X, Connelly JJ, Wang C-L, Sternglanz R. Importance of the Sir3 N terminus and its acetylation for yeast transcriptional silencing. Genetics. 2004 Sep;168(1):547-51.

43. Asaumi M, lijima K, Sumioka A, lijima-Ando K, Kirino Y, Nakaya T, et al. Interaction of $\mathrm{N}$-terminal acetyltransferase with the cytoplasmic domain of beta-amyloid precursor protein and its effect on A beta secretion. J Biochem. 2005 Feb;137(2):147-55.

44. Myklebust LM, Van Damme P, Støve SI, Dörfel MJ, Abboud A, Kalvik TV, et al. Biochemical and cellular analysis of Ogden syndrome reveals downstream Nt-acetylation defects. Hum Mol Genet. 2015 Apr 1;24(7):1956-76.

45. Behnia R, Panic B, Whyte JRC, Munro S. Targeting of the Arf-like GTPase Arl3p to the Golgi requires N-terminal acetylation and the membrane protein Sys1p. Nat Cell Biol. 2004 May;6(5):405-13.

46. Setty SRG, Strochlic TI, Tong AHY, Boone C, Burd CG. Golgi targeting of ARFlike GTPase Arl3p requires its Nalpha-acetylation and the integral membrane protein Sys1p. Nat Cell Biol. 2004 May;6(5):414-9.

47. Behnia R, Barr FA, Flanagan JJ, Barlowe C, Munro S. The yeast orthologue of GRASP65 forms a complex with a coiled-coil protein that contributes to ER to Golgi traffic. J Cell Biol. 2007 Jan 29;176(3):255-61. 
48. Forte GMA, Pool MR, Stirling CJ. N-terminal acetylation inhibits protein targeting to the endoplasmic reticulum. PLoS Biol. 2011 May;9(5):e1001073.

49. Scott DC, Monda JK, Bennett EJ, Harper JW, Schulman BA. N-terminal acetylation acts as an avidity enhancer within an interconnected multiprotein complex. Science. 2011 Nov 4;334(6056):674-8.

50. Monda JK, Scott DC, Miller DJ, Lydeard J, King D, Harper JW, et al. Structural conservation of distinctive $\mathrm{N}$-terminal acetylation-dependent interactions across a family of mammalian NEDD8 ligation enzymes. Structure. 2013 Jan

51. Polevoda B, Cardillo TS, Doyle TC, Bedi GS, Sherman F. Nat3p and Mdm20p are required for function of yeast NatB Nalpha-terminal acetyltransferase and of actin and tropomyosin. J Biol Chem. 2003 Aug 15;278(33):30686-97.

52. Coulton AT, East DA, Galinska-Rakoczy A, Lehman W, Mulvihill DP. The recruitment of acetylated and unacetylated tropomyosin to distinct actin polymers permits the discrete regulation of specific myosins in fission yeast. J Cell Sci. 2010 Oct 1;123(Pt 19):3235-43.

53. Jaillon $\mathrm{O}$, Aury J-M, Wincker P. "Changing by doubling", the impact of Whole Genome Duplications in the evolution of eukaryotes. C R Biol. 2009 Mar;332(2-3):241-53.

54. Henry CA, Urban MK, Dill KK, Merlie JP, Page MF, Kimmel CB, et al. Two linked hairy/Enhancer of split-related zebrafish genes, herl and her7, function together to refine alternating somite boundaries. Development. 2002 Aug;129(15):3693-704.

55. Henry CA, McNulty IM, Durst WA, Munchel SE, Amacher SL. Interactions between muscle fibers and segment boundaries in zebrafish. Dev Biol. 2005 Nov 15;287(2):346-60.

\section{Figure Captions}

Fig1. SiRNA screen.

Naa15 act as a fusion inhibitor in c2c12 myoblast. c2c12-MCK:GFP Cell cultures

461 transfected with anti-Naa15 siRNA and stained with DAPI (blue) present more nuclei in

462 differentiated (GFP+) cells (A). 1B. The fusion index of the GFP+ cells is significantly higher

463 in cultures treated with the anti-Naa15 siRNA compared to control cell cultures. 1C. The

464 mean number of cells including more than 10 nuclei after 7 days of differentiation is five 
465 times higher in cell cultures treated with anti-Naal5 siRNA compared to control cell cultures.

466 No significant difference is observed in the number of small myotubes $(<4$ nuclei). QPCR

467 experiment show that, siRNA treatment reduces to half the Naa15 expression (D). The

468 expression of Myogenin (MyoG) is not affected by the anti-naa15 siRNA transfection (E).

469 Different letters indicate significant differences between groups (t-test $\mathrm{p}<0.05)$. Values

470 represent means $\pm \mathrm{SD}(\mathrm{N}=4)$. Blue and red curves and histograms represent results of cell

471 cultures treated by 2 different siRNA, control group is represented by green curves and

472 histograms.

Fig2. Naa15 orthologous genes in zebrafish.

474 The evolutionary history of NAA15 related proteins was inferred by using the maximum

475 likelihood method based on the JTT matrix-based model. The bootstrap value calculated out

476 of 500 replicates is indicated for each node. A discrete gamma distribution was used to model

477 evolutionary rate differences among sites. Naa15 duplicate into naa15a and naal5b during

478 the Teleost Genome Duplication (TGD).

$479 \quad$ Fig3. naa15a and naa15b are expressed in somite at $24 \mathrm{hpf}$.

$480 \quad n a a 15 a$ and naal5b expression in 24 hpf zebrafish embryos. naal5a is strongly expressed

481 in somites as well as in eyes and midbrain (left panel). naa15b is also expressed in somite,

482 eyes and midbrain but the signal appears weaker (right panel).

483 Fig4. Naa15 knockdown induce curvature of the body.

484 Naa15 KD induces curvature of the body. Most of the eggs injected with anti-naa15

485 morpholino gave rise to embryos with curvature of the trunk and reduction of body size.

486 Injection of mismatch morpholino do not induce apparent phenotype. The majority of the 
487 naal5a morphants presents reduction of body size and moderate curvature of the trunk

488 (bottom left) whereas most of the naa15b morphant presents greater curvature (bottom right).

489 Fig5. Naa15 knockdown induce myotome defect.

490 naal5 KD induces myotomal boundary defects. The embryos were stained with TOPRO 3

491 (nuclei in red) and WGA-alexa488 (plasma membranes and myotome boundaries in green).

492 Confocal microscopy of the group 1 and 2 embryos showed a loss of the classical chevron

493 shaped segments with a lack or reduction of the horizontal myoseptum (asterisk) and

494 interruption of myotome boundaries along the dorso/ventral and medio/lateral axis (arrow).

495 Few myofibres stretch out within two somites/myotomes dues to interruption in somite

496 boundaries (arrow head).

497 Fig6. Naa15 knockdown induce defect in the myosine organization.

498 naa15 KD induce defect in myosin organisation. Confocal microscopy of morphants after

499 staining with TOPRO 3 (nuclei in blue), WGA-alexa488 (plasma membranes and myotome

500 boundaries in green) (panel A and B), and immunocytofluoresence staining with an antibody

501 against myosin heavy chain (MF20, myosin in red) (panel C and D), revealed a

502 disorganization of the myosin localization in the naa15 morphants (D) as compared to

503 embryos injected with naal5 mismatch naal5a morpholino (C). Some long myofibres project

504 beyond the intersegmental boundaries (white circle). 
A

B

$500 \mu \mathrm{m}$ 

MO Naa15a scramble control

MO Naa15a

A

B

$$
\text { C }
$$

E
D

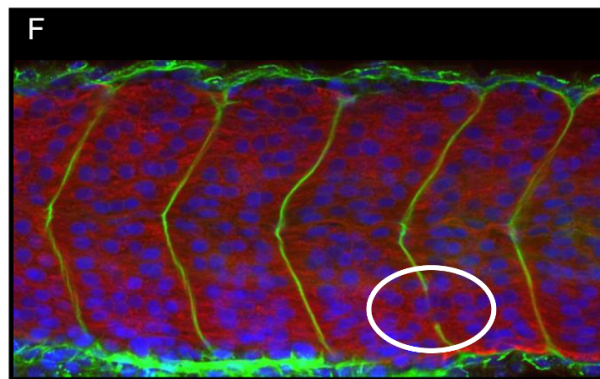

